\title{
Distribución y abundancia de la rana de invernadero, Eleutherodactylus planirostris (Cope, 1862), en Playa del Carmen, Quintana Roo, México
}

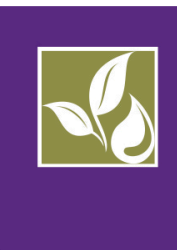

Recibido: 18/01/2016 · Aceptado: 16/03/2016

Andrés Abel Gómez-Salazar Departamento de Ingeniería Quimica y Bioquímica Instituto Tecnológico de Chetumal José Rogelio Cedeño-Vázquez* Departamento de Sistemática y Ecología Acuática

El Colegio de la Frontera Sur

\section{Resumen}

Con base en registros auditivos, se presentan los resultados del primer estudio sobre distribución y abundancia de la rana de invernadero (Eleutherodactylus planirostris) en el Caribe mexicano. Su población se encuentra bien distribuida (69\% de los sitios muestreados) en la mancha urbana de Playa del Carmen y su abundancia (tasa de encuentro promedio $=20.3$ ind. $/ \mathrm{km}$ ) indica que está bien establecida. Se espera que con los resultados obtenidos se generen acciones para el control y manejo de esta especie invasora; no obstante, es necesario ampliar los muestreos hacia la periferia de la ciudad, en sitios con vegetación conservada, para descartar la posibilidad de que la distribución actual de la especie haya sobrepasado la mancha urbana.

Palabras clave: Caribe mexicano, abundancia, distribución, especie invasora, rana de invernadero.

*Correos electrónicos: rcedenov@ecosur.mx, rogeliocedeno@gmail.com 


\title{
Distribution and abundance of the greenhouse frog Eleutherodactylus planirostris (Cope, 1862), in Playa del Carmen, Quintana Roo, Mexico
}

\author{
Andrés Abel Gómez-Salazar \\ Departamento de Ingeniería Quimica y Bioquímica \\ Instituto Tecnológico de Chetumal \\ José Rogelio Cedeño-Vázquez* \\ Departamento de Sistemática y Ecología Acuática \\ El Colegio de la Frontera Sur
}

\begin{abstract}
Based on acoustic records the first results on the distribution and abundance of the greenhouse frog (Eleutherodactylus planirotris) in the Mexican Caribbean are presented. An abundant (average encounter rate $=20.3$ ind. $/ \mathrm{km}$ ) and well established (occurred in $69 \%$ of the surveyed sites) population was detected in the urban area of the city of Playa del Carmen. It is hoped that these results induce actions for the control and management of this invasive species; however, it is necessary to extend the study to the periphery of the city, in places with preserved vegetation, to rule out the possibility that the current distribution of the species has already exceeded the urban area.
\end{abstract}

KEY words: Mexican Caribbean, abundance, distribution, greenhouse frog, invasive species.

*E-mail: rcedenov@ecosur.mx, rogeliocedeno@gmail.com 


\section{Introducción}

Las especies exóticas o introducidas son aquellas que se encuentran fuera de su área de distribución original o nativa, como consecuencia de su transportación intencional o accidental por medios humanos. Cuando esas especies llegan a establecerse, se alimentan, compiten por alimento e hibridan con especies nativas, transforman y destruyen el hábitat y además pueden portar enfermedades y parásitos transmisibles, capaces de enfermar y exterminar poblaciones y especies nativas enteras; sin embrago, los resultados pueden no ser reconocibles sino hasta varios años después de la introducción (Álvarez-Romero, Medellín, Oliveras de Ita, Gómez de Silva y Sánchez, 2008). Por estas razones es necesario diagnosticar oportunamente la situación de las poblaciones de especies de reciente invasión a fin de implementar acciones tempranas para su erradicación o control. En el caso de México, las acciones a emprender deben enmarcarse dentro de la Estrategia Nacional sobre Especies Invasoras elaborada por el Comité Asesor Nacional sobre Especies Invasoras (2010).

Respecto a los anfibios exóticos, se ha reconocido al ser humano como un agente causal en la introducción de la rana de invernadero Eleutherodactylus planirostris (figura 1), un anuro pequeño de $16-32 \mathrm{~mm}$ de longitud hocicocloaca (LHC) (Olson, Beard y Pitt, 2012), nativo de las Indias occidentales del mar Caribe (Cuba, Bahamas y las Islas Caimán) (Beard, Olson y Pitt, 2011).

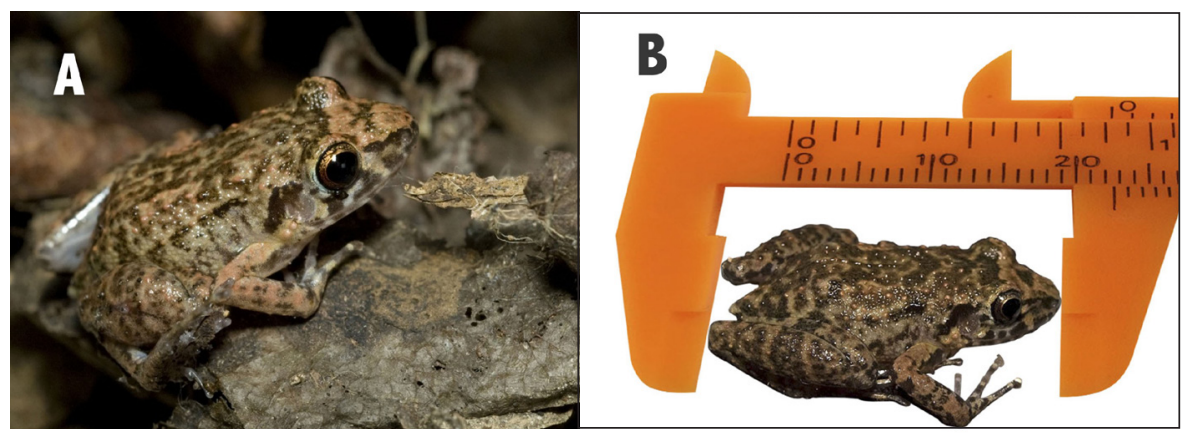

FigURA 1. Ejemplares adultos de Eleutherodactylus planirostris, colectados en la ciudad de Playa del Carmen, Quintana Roo, México. Fotografías de Humberto Bahena-Basave (A) y Javier González-Vázquez (B) 
Esta rana tiene desarrollo directo; es decir, del huevo eclosionan las crías con la misma forma de los adultos, sin haber pasado por una fase larvaria. Sus puestas contienen de 3 a 26 huevos que son depositados en el suelo húmedo, a menudo dentro de una pequeña depresión excavada por la hembra, o bien bajo vegetación en estado de descomposición, bajo la hojarasca acumulada en macetas con plantas, rocas, ladrillos o escombros; la eclosión ocurre al transcurrir dos semanas (Pough, Stewart y Thomas, 1977; Díaz y Cádiz, 2008). Es activa durante la noche y primeras horas de la mañana, sobre todo durante los meses de lluvia; se le puede encontrar en sitios húmedos, por lo general bajo montículos de hojarasca, pero puede prosperar asimismo en áreas urbanas, ocupando espacios como depósitos de chatarra, invernaderos, viveros, jardines, registros o contenedores de agua (Christy, Clark, Gee II, Vice, D., Vice, D. S., Warner, Tyrrell, Rodda, y Savidge, 2007; Díaz y Cádiz, 2008). Los machos vocalizan principalmente sobre el suelo, ocultos en algun sustrato, aunque también pueden hacerlo desde las hojas, tallos y ramas ubicados a menos de un metro de altura (Díaz y Cádiz, 2008). Su vocalización consiste en una compleja secuencia de señales donde se alternan llamadas de una, dos y tres notas, y a veces trinos de 11-13 notas, que pueden durar más de un segundo; las llamadas más frecuentes son las de dos notas, con una duración de 38-94.3 milisegundos (ms); las de una nota suelen ser cortas (6-7 ms), mientras que las de tres notas duran 117-191 ms (Díaz y Cádiz, 2008). Se alimenta de pequeños invertebrados que habitan en la hojarasca (Olson, Bread, Koons y Pitt 2012).

Debido a sus características ecológicas y a su forma de reproducción, esta rana ha sido introducida extensamente desde hace un siglo, fuera de su ámbito de distribución nativa (figura 2). Las actividades humanas han contribuido de manera significativa en este proceso a través del comercio internacional de plantas de ornato y materiales de jardinería (Christy, Savidge y Rodda, 2007; Heinicke, Díaz y Hedges, 2011; Villa, 2015). La gran adaptabilidad que tiene esta especie y el potencial para colonizar otras áreas e incrementar sus poblaciones rápidamente, representa una amenaza para la fauna nativa. Según Beard, Olson y Pitt (2011), la introducción de esta rana podría constituir una amenaza potencial para algunas especies de invertebrados terrestres, al incorporarse estos a su dieta, o para especies amenazadas al iniciarse una competencia por el alimento. Además se sabe que estas ranas pueden ser un vector de los huevos de nematodos parásitos de plantas (Beard et al., 2011; Kraus y Campbell, 2002). 




Fuente: Heinicke, Díaz y Hedges (2011).

FIgURA 2. Mapa que muestra la distribucion nativa de Eleutherodactylus planirostris en islas del mar Caribe (delimitada por lineas discontinuas) y las áreas donde se ha registrado su introducción desde 1863. Su presencia en Cayos Miskito, Nicaragua, ha sido confirmada por Villa (2015)

En México, E. planirostris fue reportada por primera vez en el estado de Veracruz (Schwartz, 1974). Recientemente, Cedeño-Vázquez, González-Vázquez, Martínez-Arce y Canseco-Márquez (2014), basados en la identificación morfológica y en el análisis molecular de ejemplares colectados en 2010 en la mancha urbana de la ciudad de Playa del Carmen, reportaron el primer registro de la especie para el Caribe mexicano, y sugieren la necesidad de conocer el estado de su invasión en esta demarcación. Tal situación motivó realizar este estudio, con el propósito de determinar la distribución y abundancia de la especie en esta ciudad.

\section{Métodos}

De agosto a noviembre de 2014 se efectuaron muestreos nocturnos en la ciudad de Playa del Carmen (figura 3), seguidos de periodos de lluvia intensa, intermitente o chubascos esporádicos, para contar con la humedad suficiente y así escuchar vocalizar a E. planirostris durante los recorridos. 


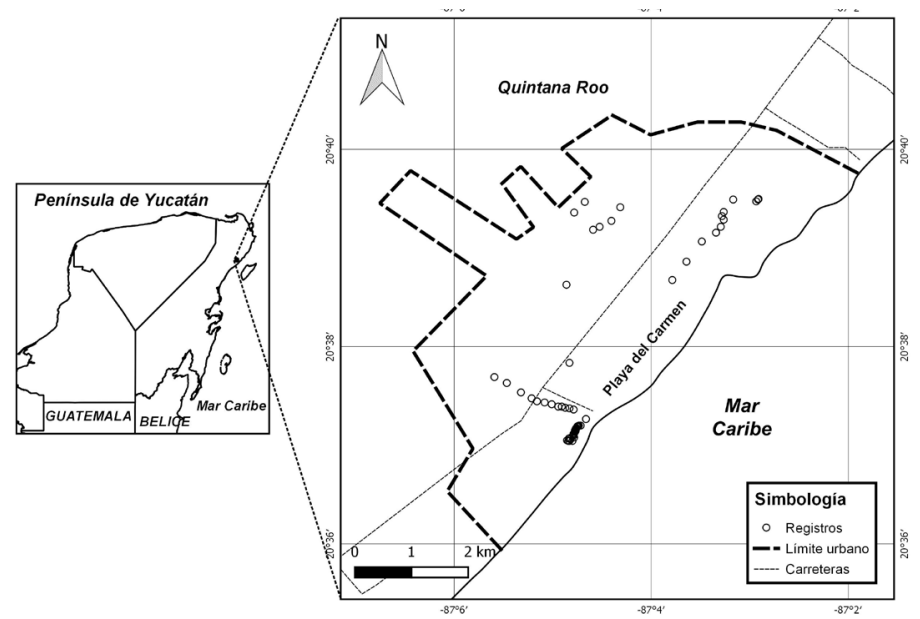

Figura 3. Mapa del área de estudio. Se muestra la distribución de Eleutherodactylus planirostris en la mancha urbana de Playa del Carmen, Quintana Roo, México.

Los muestreos se iniciaron a las 21:00 horas y concluyeron hasta las 6:00 horas, horario generalizado, en el cual vocaliza la mayoría de las especies de Eleutherodactylus caribeños (Díaz y Cádiz, 2008). La técnica de muestreo utilizada fue la de bandas o transectos para registros auditivos, que se fundamenta en las vocalizaciones emitidas por los machos adultos durante la época reproductiva, las cuales son específicas para cada especie (Rueda, Castro y Cortez, 2006).

Se tuvo un entrenamiento previo para el reconocimiento de las vocalizaciones de E. planirostris, el cual consistió en escuchar grabaciones de varios individuos, disponibles en internet, y de esta forma no confundirlas con las vocalizaciones de otras especies de ranas que pudieran estar presentes en el área de estudio.

Se establecieron 22 transectos de muestreo de $500 \mathrm{~m}$ de longitud cada uno sobre las vialidades de cuatro zonas de la mancha urbana, en función de su accesibilidad y con el propósito de cubrir la mayor parte de la ciudad. Los recorridos se hicieron a pie, marcando cada sitio de registro con un Global Positioning System (GPS). Estos datos se anotaron en la bitácora y en las tablas de registro de campo para generar el mapa de distribución de la especie en el área de estudio.

Los registros auditivos se realizaron al momento en que los machos que se encontraron vocalizaron durante los recorridos en los transectos de muestreo, 
por medio de una grabadora reportera. Para ello se efectuaban grabaciones de diversos lapsos de duración con base en la actividad de vocalizacion: tres minutos cuando la vocalizacion no era inducida, cinco cuando fueron estimulados con vocalizaciones grabadas, y diez cuando los machos no respondían a las grabaciones de vocalizaciones, aunque estuvieran presentes. En algunos sitios con abundante vegetación, al aproximarse las ranas dejaban de vocalizar y no se podía realizar un registro auditivo preciso del número de individuos presentes, situación que se resolvía reproduciendo la grabación de un individuo vocalizando, para inducir a ello.

Para determinar la abundancia en cada transecto, se establecieron cinco categorías, de acuerdo con Rice, Waddle, Crockett, Bugbee, Jeffery, y Percival (2007): a) 1 individuo, b) 2-5 individuos, c) 6-10 individuos y d) más de 10 individuos. Cabe mencionar que esta técnica se utilizó también cuando la zona de muestreo presentaba características que no permitían encontrar a la especie por medio de registro visual (por ejemplo maleza $\geq 0.5 \mathrm{~m}$ de altura, abundante escombro), donde fue muy común hallar a la especie vocalizando, pero no podía ser observada, debido a su reducido tamaño y características morfológicas, que le permiten camuflarse en el entorno.

La abundancia se determinó con un índice de densidad, mediante el registro de la tasa de encuentro, la cual puede obtenerse si se registra el tiempo invertido en cada muestreo y el número de individuos detectados, al dividir este último entre las horas invertidas en el muestreo (Rueda et al., 2006). También puede extraerse mediante los registros obtenidos por unidad de superficie (Olson, Beard y Pitt, 2012). En este estudio, por las dificultades para muestrear áreas específicas en la mancha urbana, en lugar de la superficie, se utilizó la distancia recorrida (No. ind./km).

\section{Resultados}

\section{Distribución}

Se registró un total de 223 individuos de Eleutherodactylus planirostris, en 15 de los 22 transectos muestreados, lo cual denota su presencia en $69 \%$ del área cubierta en la mancha urbana de Playa del Carmen (figura 3). 


\section{Abundancia}

La tasa de encuentro promedio registrada fue de 20 ind./km (rango: 3-33). La mayoría de los sitios muestreados son muy similares, debido a que es una ciudad con escasas áreas verdes y altamente urbanizada. Durante los muestreos se logró observar cómo la especie exhibe mayor preferencia por sitios perturbados, respecto a sitios más conservados, o áreas verdes colindantes con el espacio urbanizado.

\section{Discusión}

La probabilidad de detección de Eleutherodactylus planirostris en el área de estudio fue más alta (69\%), comparada con la reportada previamente por Rice et al. (2007) en Biscayne National Park (38\%) y Olson et al. (2012) en la isla de Hawai (41\%), áreas donde ambos estudios demostraron el establecimiento exitoso de la especie. Cabe mencionar que los últimos muestreos en este estudio se realizaron durante la entrada de un frente frío, con temperaturas por debajo de los $20^{\circ} \mathrm{C}$, situación que no impidió su actividad, como también se ha observado en los Cayos de Florida, Estados Unidos (Rice et al., 2007; Heinicke et al., 2011).

La probabilidad de detección de la especie durante el periodo de muestreo aumentó entre octubre y noviembre, lo cual confirma que tiene una mayor actividad de vocalización durante la temporada de mayor precipitación, asociada a una mayor humedad relativa y cobertura de nubosidad (Goin, 1947; Meshaka y Layne, 2005; Weir, Royle, Nanajappa, y Jung, 2005; Rice et al., 2007; Olson et al., 2012).

Las áreas verdes ocupadas por E. planirostris y zonas cercanas al área donde fue detectada por primera vez en Playa del Carmen presentan características similares a las descritas por Olson, Beard y Pitt (2012), donde prevalece gran cantidad de hojarasca, maceteros e instalaciones de toma de agua potable. No obstante, E. planirostris ha colonizado tanto áreas verdes como urbanas, por ejemplo la isla de Guam (Stewart y Martin, 1980) y el presente estudio. Los individuos registrados en Playa del Carmen muestran una mayor preferencia por los sitios perturbados y áreas con mayor impacto de actividades humanas, lo cual concuerda con lo expuesto por Beard et al. (2011). 
Cabe mencionar que se registró una mayor abundancia de E. planirostris en los sitios cercanos al área donde la especie fue reportada por primera vez en 2010 (Cedeño-Vázquez et al., 2014), por lo cual no se descarta la posibilidad de que en la misma se localice el punto de su introducción en el Caribe mexicano. Playa del Carmen es una ciudad predominantemente turística, lo que demanda la introducción de plantas exóticas para decorar las instalaciones hoteleras, factor que contribuye a la translocación de esta rana fuera de su área de distribución nativa, como consecuencia de la exportación de plantas tropicales ornamentales que le sirven como medio de transporte desde su área de origen (Kraus y Campbell, 2002; Pough, Stewart y Thomas, 1977; Villa, 2015).

Las condiciones ambientales de la mancha urbana de Playa del Carmen ofrecen suficiente humedad en los microambientes ocupados por la especie, mismas que le han permitido establecerse de manera exitosa. Resulta importante señalar que entre 2011 y 2013 se colectaron ejemplares de crías y juveniles de esta especie dentro de la ciudad, y se ingresaron a la Colección Herpetológica de El Colegio de la Frontera Sur, Unidad Chetumal (ECO-CH-H 2949-50, 3018-3020), lo cual es un indicador de que la población se encuentra reproductivamente activa.

Es necesario continuar estudiando la población de esta rana y ampliar los muestreos hacia la periferia de la ciudad, en sitios con vegetación conservada, para descartar la posibilidad de que su distribución actual haya rebasado la mancha urbana. Los futuros análisis sobre su dieta podrían revelar si las poblaciones de algunos invertebrados considerados en alguna categoría de riesgo pudieran estarse viendo afectadas.

Por otra parte, cabe apuntar que recién se detectó una población de E. planirostris en la ciudad de Cancún (García-Balderas, Cedeño-Vázquez y MinerosRamírez, 2016) y en la isla de Cozumel (Pavón-Vázquez, Gray, White, GarcíaVázquez y Harrison, 2016) cuya presencia muy posiblemente se deba al traslado de plantas decorativas procedentes de Playa del Carmen, lo cual podría demostrarse mediante análisis moleculares de las poblaciones. Ante este panorama es menester documentar el estado de la población en términos de abundancia, estructura y distribución en estos destinos turísticos, además de realizar muestreos para detectar su posible presencia en las otras ciudades de Quintana Roo, a fin de contar con un diagnóstico completo de la situación de esta rana en el Caribe mexicano. 


\section{Conclusiones}

La presencia de Eleutherodactylus planirostris en 15 de los 22 transectos muestreados (69\%) y su abundancia (223 registros, 20.3 ind./km en promedio) demuestran que existe una población establecida en la mancha urbana de Playa del Carmen. Las características de la mayoría de los sitios muestreados son muy similares, debido a que la ciudad está muy urbanizada y casi no cuenta con áreas verdes, de ahí que la especie se haya observado con mayor frecuencia en torno a edificaciones con presencia humana, en comparación con las áreas verdes colindantes.

\section{Agradecimientos}

Los autores agradecen al biólogo Javier González Vázquez, responsable del Aviario Xamán Ha, las facilidades otorgadas en Playa del Carmen para la realización de los muestreos, a Jhony Hernández Polanco por el acompañamiento durante los recorridos, y a Janneth A. Padilla Saldívar por la elaboración del mapa del área de estudio. Las observaciones de dos revisores anónimos ayudaron a mejorar el contenido del manuscrito.

\section{Fuentes consultadas}

Álvarez-Romero, J. G., Medellín, R.A., Oliveras de Ita, A., Gómez de Silva, H. y Sánchez, O. (2008). Animales exóticos en México: una amenaza para la biodiversidad. México: Comisión Nacional para el Conocimiento y Uso de la Biodiversidad/Instituto de Ecología-Universidad Nacional Autónoma de México/Secretaría de Medio Ambiente y Recursos Naturales.

Beard, K. H., Olson, C. A., Pitt, W. C. (2011). Biology and Impacts of Pacific Island Invasive Species: 8. Eleutherodactylus planirostris, the Greenhouse Frog (Anura: Eleutherodactylidae). Wildland Resources Faculty Publications, Utah State University. Recuperado de http://digitalcommons.usu. edu/cgi/viewcontent.cgi? article $=2023 \&$ context $=$ wild_facpub.

Cedeño-Vázquez, J. R., González-Vázquez, J., Martínez-Arce, A. y Canseco-Márquez, L. (2014). First record of the invasive greenhouse frog 
(Eleutherodactylus planirostris) in the Mexican Caribbean. Revista Mexicana de Biodiversidad, 85(2), 650-653.

Christy, M. T., Savidge, J. A. y Rodda, G. H. (2007). Multiple pathways for invasion of anurans on a Pacific island. Diversity and Distributions, 13, 598-607.

Christy, M. T., Clark, C. S., Gee II, D. E., Vice, D., Vice, D. S., Warner, M. P., Tyrrell, C. L., Rodda, G. H. y Savidge, J. A. (2007). Recent records of alien anurans on the Pacific island of Guam. Pacific Science, 61(4), 469-483.

Comité Asesor Nacional sobre Especies Invasoras (2010). Estrategia Nacional sobre Especies Invasoras en México: prevención, control y erradicación. México: Comisión Nacional para el Conocimiento y Uso de la Biodiversidad/Secretaría de Medio Ambiente y Recursos Naturales.

Díaz, L. M. y Cádiz, A. (2008). Abc Taxa: Guía taxonómica de los anfibios de Cuba. Vol. 4. Bruselas: Royal Belgium Institute of Natural Sciences.

García-Balderas, C. M., Cedeño-Vázquez, J. R. y Mineros-Ramírez, R. (2016). Eleutherodactylus planirostris. Distribution note. Mesoamerican Herpetology, 3(1), 173.

Goin, C. J. (1947). Studies on the life history of Eleutherodactylus ricordii planirostris (Cope) in Florida, with special reference to the local distribution of an allelomorphic color pattern. Gainesville: University of Florida Press.

Heinicke, M. P., Díaz, L. M. y Hedges, S. B. (2011). Origin of invasive Florida frogs traced to Cuba. Biology Letters, 7, 407-410.

Kraus, F. y Campbell, E. W. I. (2002). Human-mediated escalation of a formerly eradicable problem: The invasion of Caribbean frogs in the Hawaiian Islands. Biological Invasions, 4, 327-332.

Meshaka, W. E. Jr. y Layne, J. N. (2005). Habitat relationships and seasonal activity of the greenhouse frog (Eleutherodactylus planirostris) in southern Florida. Florida Scientist, 68, 35-43.

Olson, C. A., Beard, K. H. y Pitt, W. C. (2012). Biology and Impacts of Pacific Island Invasive Species. 8. Eleutherodactylus planirostris, the Greenhouse Frog (Anura: Eleutherodactylidae). Pacific Science, 66(3), 255-270. Recuperado de https://www.aphis.usda.gov/wildlife_damage/ nwrc/publications/12pubs/pitt121.pdf. 
Olson, C. A., Bread, K. H., Koons, D. N. y Pitt, W.C. (2012). Detection probabilities of two introduced frogs in Hawaii: implications for assessing nonnative species distributions. Biological Invasions, 14, 889-900.

Pavón-Vázquez, C. J., Gray, L. N., White, B. A. García-Vázquez U. O. y Harrison, A. S. (2016). Distribution notes. First records for Cozumel Island, Quintana Roo, Mexico: Eleutherodactylus planirostris (Anura: Eleutherodactylidae), Trachycephalus typhonius (Anura: Hylidae), and Indotyphlops braminus (Squamata: Typhlopidae). Mesomerican Herpetology, 3 (2), 531-533.

Pough, F. H., Stewart, M. M. y Thomas, R. G. (1977). Physiological basis of habitat partitioning in Jamaican Eleutherodactylus. Oecologia, 27, 285-293.

Rice, K. G., Waddle, J. H., Crockett, M. E., Bugbee, C. D., Jeffery, B. M. y Percival, H. F. (2007). Herpetofaunal inventories of the National Parks of South Florida and the Caribbean: Volume IV. Biscayne National Park. Reston: U. S. Geological Survey.

Rueda, J. V., Castro, F. y Cortez, C. (2006). Técnicas para el inventario y muestreo de anfibios: Una compilación. En A. Angulo, J V. Rueda-Almonacid, J. V. Rodríguez-Mahecha y E. La Marca (eds.), Técnicas de inventario y monitoreo para los anfibios de la Región Andina. Bogota: Conservación Internacional, Serie Manuales para la Conservación 2. Recuperado de http://www.amphibians.org/wp-content/uploads/2013/07/ Monitoreo-de-anfibios-baja-final.pdf [2016,14 de enero].

Schwartz, A. (1974). Eleutherodactylus planirostris. Catalogue of American Amphibians and Reptiles, 154, 1-4.

Stewart, M. M. y Martin, G. E. (1980). Coconut-husk piles - a unique habitat for Jamaican terrestrial frogs. Biotropica, 12,107-116.

Villa, J. (2015). Las ranitas de Cayos Miskitos. Revista de Temas Nicaragüenses, $89,6-22$.

Weir, L. A., Royle, J. A., Nanajappa, P. y Jung, R. E. (2005). Modeling anuran detection and site occupancy on North American Amphibian Monitoring Program (NAAMP) routes in Maryland. Journal of Herpetology, 39, 627-638. 